\author{
Joseph B. Sidowski
}

\title{
Products
}

\section{Telemetry Receiver}

Dual-channel telemetry receivers are available from EKEG Electronics. Various channel combinations are obtainable including EKG and respiration, and fetal ECG and interuterine pressure. Appropriate voltage levels and scope output are available from each channel. Receiver tuning range is $87.108 \mathrm{MHz}$.

EKEG Electronics Co., Ltd.

Box 310

Station A

Vancouver 1, B.C., Canada

\section{Multiplexer}

The TDX-2 multiplexer will speed the mixing of data at rates of 300,150 , 134.49 , and/or $110 \mathrm{bits} / \mathrm{sec}$. Capable of handling up to 88 channels, the unit is "data transparent" and will transmit all combinations of seven- or eight-bit data characters. The TDX-2 can be used as the front-end multiplexer/demultiplexer for a large machine.

Rixon Electronics, Inc.

2120 Industrial Parkway

Silver Spring, Maryland 20904

(301) 622-2121

\section{Mag Tape Units}

A new line of IBM-compatible magnetic tape units, Models 8551 (seven-track) and 8552 (nine-track), feature triple-density reading and writing $(200,556$, and $800 \mathrm{bps})$, the automatic generation of interblock gaps, longitudinal redundancy checking code, and cyclical redundancy checking. Incremental mode writing covers $0-1,000$ characters/sec at any density; incremental reading covers the range of 0-500 characters/sec. The machines can shuttle back and forth over a single character when reading incrementally, providing character buffering. Standard tape speed in the synchronous mode is $30 \mathrm{in} / \mathrm{sec}$ with bidirectional operation within standard interblock gapping. The units operate at speeds of 24,000 characters $/ \mathrm{sec}$ at $800 \mathrm{bpi}$. Model 8551 is priced at $\$ 6,625$; the 8552 is $\$ 6,900$.

\footnotetext{
Datron Corporation

179 W. Rock Road

Norwalk, Connecticut 06851

(203) $847-9669$
}

\section{Minicomputer Price Reduction}

The Computer Automation, Inc., Model 216 minicomputer has been reduced in price from $\$ 12,890$ to $\$ 7,990$. The Model 216 has a $4,096 \times 16$ bit memory expandable to $32 \mathrm{~K}$ and over 122 instructions. Other characteristics include: $42 \mu \mathrm{sec}$ software multiply, hardware indexing, 500,000-bytes/sec input-output, three priority interrupts with unlimited expansion, immediate and compare instructions, and direct memory access.

Computer Automation, Inc.

895 West 16th Street

Newport Beach, California 92660

\section{Digital Printer}

The Model 1454 Digital Printer accepts $1-2-4.8,1-2-2-4,1-2-4-2 \mathrm{BCD}$, and 10-line inputs in any combination, and is compatible with vacuum-tube, transistor, and IC logic levels. Use of individual printed-circuit column modules permits data columns to be spaced in accordance with requirements. Maximum printed speed is three lines per second. A floating decimal point in the same column as the selected numerical data and automatic suppression of insignificant zeros are available as options. Price: $\$ 1,195$.

Beckman Instruments, Inc.

Electronics Instruments Division

2200 Wright Avenue

Richmond, California 94804

\section{Multiplex-Coupler}

The Model 5010 multiplex-coupler allows measurements to be processed in ASCII code and provides the interface between digital instruments and computers or $\mathrm{I}-\mathrm{O}$ devices. The 5010 accepts four-line bed inputs from such instruments as counters, digital multimeters, time-code generators, and A-to-D converters, and translates the data into serially coded information for direct handling by a computer or ASR-33 Teletype. Outputs are compatible with magnetic tapes, punch paper tapes, IBM punch cards, and IBM Selectric typewriter Model 735. Price of the terminal with one input card is $\$ 1,350$.

Electrodata Corporation

Concord, California 94522

\section{Desktop Strip Printer}

The Model 3064-C desktop strip printer prints 30 characters per second, yet is only $8 \mathrm{in}$. long and weighs $4 \mathrm{lbs}$. Sound attenuation is low and easy readout is provided by characters nearly $1 / 8 \mathrm{in}$. high (nine/inch) in a font of 64 characters-ASCII full alphanumeric plus symbols subset. Logic levels are compatible with IC logic elements used in data communications systems and devices. The unit's active input states minimize the logic required to implement operating interface with a remote computer. Price: $\$ 55$.

Dataline, Inc.

King of Prussia

Pennsylvania 19406

\section{Sound-Level Meter}

Radio Shack is marketing a sound-level meter (SLM) for only $\$ 39.95$. The Realistic SLM is a hand-held nugged solid-state instrument with a usable range of $65.100 \mathrm{~dB}$ for measuring sound intensities. A mode control switch provides power to the meter whenever it is in either the FAST or SLOW positions. The output jack allows the instrument to be used as a microphone. Electrical equilizers maintain the frequency response between $40 \mathrm{~Hz}$ and $8,500 \mathrm{~Hz} \pm 2 \mathrm{~dB}$. High-impedance headphones can be connected to the output jack as can an oscilloscope or a distortion analyzer. The cited specifications are as follows. Range: $65-80$, 75-90, 85-100, 96-110 dB. Accuracy: $\pm 2 \mathrm{~dB}$ at $74-\mathrm{dB}$ sound pressure. Output: $1 \mathrm{~V}$ at $1,000 \mathrm{~Hz}$. Output impedance: 10,000 ohms minimum. Output gain: $72 \pm 3 \mathrm{~dB}$ at $1,000 \mathrm{~Hz}$. Output gain: less than $2 \%$ at $1,000-\mathrm{Hz}, 0.5 . \mathrm{V}$ output.

Hirsch-Houck Laboratories evaluated the Realistic unit by checking it against a calibrated Altec microphone. The Realistic SLM read slightly low (3-5 dB) at 70,90 , and $100 \mathrm{~dB}$, but a screwdriver adjustment allows calibration to be set accurately. Incrementally, the Realistic was judged to be quite accurate, to within $1 \mathrm{~dB}$. Tape recordings made with the instrument as a microphone were compared with recordings made with an inexpensive dynamic mike supplied with an Ampex Model 1455 A tape recorder. Sound quality of the Realistic was adequate but not quite as clean as the Ampex mike. 
However, the output signal was found to be exceptionally high and could not be fed into the mike input of the recorder without overloading. The gain in to the line input was so high that the unit was operated at $110 \mathrm{~dB}$ sensitivity (minimum) for use within a few feet. Sounds from 20 to $30 \mathrm{ft}$ away were picked up with clarity. Overall, the Hirsch-Houck evaluation indicated that the Realistic SLM was a fine dual-purpose instrument for the price.

\section{Digital Printer}

The Victor Imperial digital printer prints eight-column figures from remote sources on either 24- or $48-\mathrm{V}$ solenoids for $\$ 335$. Ten-column capacity costs $\$ 20$ more; addition and subtraction are available for $\$ 50$.

\section{Victor Comptometer Corporation \\ Business Machines Group \\ 3900 North Rockwell Street \\ Chicago, Illinois 60618}

\section{Fetal Pulse Detector}

Minidop is a miniature ultrasonic doppler fetal pulse detector priced at $\$ 445$. The unit is easily hand held and is advertised as being capable of detecting fetal life as early as 10 weeks.

\section{Magnaflux Corporation \\ Medical Division \\ 7314 West Lawrence Avenue \\ Chicago, Illinois 60656}

\section{Briefs}

\section{Iliac IV Computer}

The new IV computer is a most powerful machine with 64 parallel processors, each with a cycle time of 50 nsec, operating under the control of a Model B-6500 computer built by Burroughs Corporation. The machine is said to be capable of simultaneously multiplying 64 pairs of 64-bit numbers in 45 nsec.

\section{Supercomputers Measured in MIPS}

A variety of supercomputers will reach the market in the early 1970s. As they do, MIPS (millions of instructions per second) will serve as a characteristic measurement. The IBM 360/195 and the Control Data Corporation 7600 both operate in the area of 15 million instructions per second, or MIPS. The CDC STAR (STring ARay) processor will operate at about 60 MIPS. This is also the speed range for the stripped-down Iliac IV (64 parallel processors rather than 265) described above. By 1975, IBM is said to be planning a 250-MIPS entry into the field. Last year a
CDC representative speculated that the next 5 years would bring a fivefold increase in instructions per second as it had in the last 5-year period. This projection would bring the CDC machine levels up to 75 MIPS. But, more recently, CDC's proposals are projected at 1,000 MIPS by 1975 (kiloMIPS), a tremendous increase. At present the Russians are believed to be at about the 0.5-MIPS level.

\section{Teach-a-Pet}

Teach-a-Pet is a new, low-cost experimental teaching unit designed to enable students to perform basic studies in animal behavior. The unit illustrated in Fig. 1 is manually operated, battery-powered, and advertised as safe for children. A guide is provided which stresses the importance of reinforcement for shaping behavior. Cost of the basic box and guide is $\$ 18.95$.

\section{Animal Technology, Inc. 6 Beck Road Arlington, Massachusetts 02174 (617) 648-3871}

\section{Instrument/Computer Interface}

A new system from Princeton Applied Research Corporation (P.A.R.) reduces real-time experimental data, then transfers the data to a remote time-shared computer. An $E$ can then conduct immediate analysis of the collected data by interacting with the computer via a Teletype terminal and phone link. The storage capacity of the large, multiprocessing system allows for the collection of huge quantitites of data for complex programs, while special-character detection circuits permit the experiment to be controlled by remote computer.

The P.A.R. Model 131 Instrument/Computer Interface System can accept data from up to 90 analog and digital instruments, such as signal averagers, lock-in amplifiers, signal correlators, polagraphic instruments, and other measuring devices. These data are processed by appropriate instrument modules.

With the Model 131, the time-sharing computer can operate in the question/response mode. In answer to the computer's requests, you can input varying parameters for the experiment to obtain new test results from the same set of data.

Output from analog experimental instruments is analog multiplexed, then converted to digital form. Output is fed to a digital multiplexer. This module converts the input to serial form for output to the teletypewriter, acoustic coupler, or data set for transmission to the computer center.

Price of a typical system consisting of an analog multiplexer, A-D converter, a system interface, and teleprinter is advertised at less than $\$ 6,200$.

\section{Telemetry}

Telemetric subcarrier bands are listed in Table 1.

\section{TV Camera Tube for "Seeing in the Dark"}

RCA has developed a TV camera tube sensitive enough to record in almost complete darkness, yet the tube can withstand bright sunlight. Evidently useful

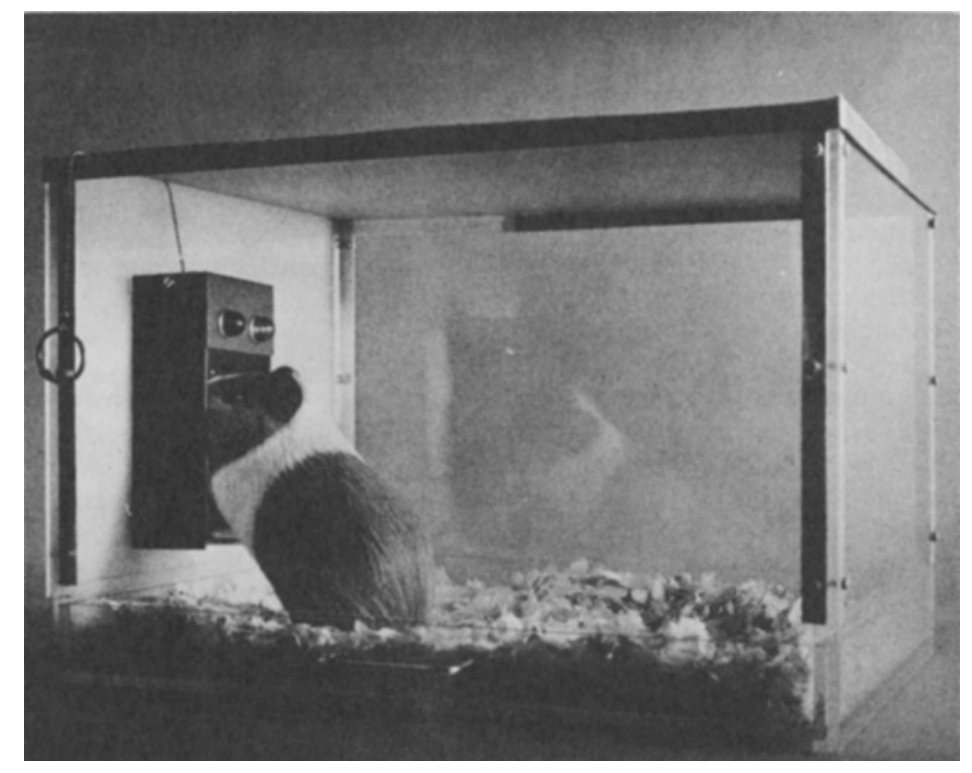

Fig. 1. Teach-a-Pet unit. 
Table 1

Telemetric Subcarrier Bands

\begin{tabular}{|c|c|c|c|c|}
\hline \multirow[b]{2}{*}{ Band } & \multirow{2}{*}{$\begin{array}{c}\text { Center } \\
\text { l:requency } \\
\text { cps }\end{array}$} & \multicolumn{2}{|c|}{$\begin{array}{l}\text { Limits } \\
\text { cps }\end{array}$} & \multirow{2}{*}{$\begin{array}{c}\text { Frequency } \\
\text { Response }\end{array}$} \\
\hline & & Upper & Lower & \\
\hline 1 & 400 & 430 & 370 & 6 \\
\hline 2 & 560 & 602 & 518 & 8 \\
\hline 3 & 730 & 785 & 675 & 11 \\
\hline 4 & 960 & 1,032 & 888 & 14 \\
\hline 5 & 1,300 & 1,398 & 1,202 & 20 \\
\hline 6 & 1,700 & 1,828 & 1,572 & 25 \\
\hline 7 & 2,300 & 2,473 & 2,127 & 35 \\
\hline 8 & 3,000 & 3,225 & 2,775 & 45 \\
\hline 9 & 3,900 & 4,193 & 3,607 & 60 \\
\hline 10 & 5,400 & 5,805 & 4,995 & 80 \\
\hline 11 & 7,350 & 7,901 & 6,799 & 110 \\
\hline 12 & 10,500 & 11,288 & 9,712 & 160 \\
\hline 13 & 14,500 & 15,888 & 13,412 & 220 \\
\hline 14 & 22,000 & 23,650 & 20,350 & 330 \\
\hline 15 & 30,000 & 32,250 & 27,750 & 450 \\
\hline 16 & 40,000 & 43,000 & 37,000 & 600 \\
\hline 17 & 52,500 & 56,440 & 48,560 & 790 \\
\hline 18 & 70,000 & 75,250 & 64,750 & 1,050 \\
\hline A & 22,000 & 25,300 & 18,700 & 660 \\
\hline B & 30,000 & 34,500 & 25,500 & 900 \\
\hline C & 40,000 & 46,000 & 34,000 & 1,200 \\
\hline D & 52,500 & 60,380 & 44,620 & 1,600 \\
\hline $\mathrm{E}$ & 70,000 & 80,500 & 59,500 & 2,100 \\
\hline
\end{tabular}

pictures can be picked up from an area illuminated with a light level equivalent to that supplied by a 100-W lamp a couple of miles away. The Silicon Intensifier Tube (SIT) contains a special electronics light amplifier and a silicon-integrated circuit target as its "retina." The tube consists of a vidicon-type scanning electron gun and image intensifier section separated by the IC target. The latter provides the gain, which results in the ultrasensitive performance at low light levels and consists of a two-dimensional array of more than 600,000 junction diodes formed in an n-type silicon wafer.

\section{Solid-State Relay}

Ebeko of Urloffen, West Germany, has developed an interesting solid-state relay that features an integral four-transistor direct-coupled amplifier. The unit employs a conventional electromagnetic input coil which, instead of operating a mechanical armature, acts to control the resistance of a Hall-effect indium-antimonide element. In turn, the Hall-effect resistor adjusts the amplifier's bias to provide output switching with one driver transistor used as the normally "off" and another as the normally "on" contact. The relay is suitable for use in $\mathrm{dc}$ and in pulsed ac circuits and has the advantages of fast, bounceless, arcless operation, virtually unlimited life, and excellent I/O isolation

\footnotetext{
Magnistors

"Magnistors" are being offered by the Hudson Corporation (Box 867 , Manchester, N.H. 03105) at unit prices
}

ranging from $\$ 3.50$ to $\$ 5$ with a minimum order of $\$ 30$. A Magnistor is a silicon planar transistor with two collectors and a single emitter. The construction is arranged so that an external magnetic field gives a lateral motion to the charge flow from the emitter. This causes a differential change in the relative collector currents. Magnistors are used as magnetic sensors in control operations.

\section{IBM's New Computer}

IBM recently introduced Models 155 and 165 of the new computer System-370. Both models have internal operating speeds two to five times faster than medium and large models of the IBM-360. Announced with System-370 was a magnetic disk-storage device that can hold up to 800 million characters. Average access time to the electronic file is $30 / 1,000$ sec. A new printer has been introduced also. The IBM 3211 printer operates at 2,000 lines per minute.

\section{Soviet Computing}

A recent report by E. E. David, Jr., of Bell Telephone Laboratories, indicates an accelerating interest in computing machinery and software in Russia. Soviet personnel involved in scientific computing were judged as highly competant and knowledgeable of the latest techniques, but there is an appreciable lag in hardware development, particularly in bulk storage devices, peripheral and $1 / 0$ devices, and circuit technology.

The best-known transistorized computer is the BESM-6, a 48-bit machine,
$64 \mathrm{~K}$-words, a $2-\mu \mathrm{sec}$ main memory that is eight-way interleaved. This computer's 32 high-speed registers are capable of up to one million instructions per second. Since no disk files are made in Russia at present, mass storage is all magnetic drum. Of course, tape units are used.

Other popular computers are the Minsk-22 and the M-220. The Minsk is typically an $8 \mathrm{~K}$-word machine with a 37-bit memory capable of up to 6,000 operations per second. The M-220 executes up to 30,000 three-address operations per second. Like most other USSR computer machinery, the M-220 is large. It occupies seven or eight fullsize equipment bays for $\mathrm{cpu}, \mathrm{I} / \mathrm{O}$ controller, and memory.

Most machines use $16 \mathrm{~K}$-word drums; mass storage devices are noticeably absent. Large core storage is unknown.

Algol is the most popular language, but a Fortran compiler is available for the BESM-6. A three-partition multiprogramming system is used in operating the machine. In spite of the high degree of centralized control, considerable incompatibility exists between Russian machines. And octal machine code is still being used for much of the programming.

The Institute for Computing Sciences in Novosibirk supplies services to all 20 research institutes in the academic city. Approximately 1,000 of the institute's users are claimed to do their own programming. The BESM-6 runs three shifts per day, with turn-around time approximately 1 day. Privileged users get immediate turn-around. There are 15 key-punch operators on two shifts. Programmers use card inputs and tapes for data. No graphic outputs are noticeable, although an experimental CRT display has been built.

Users of the BESM-6 are regularly charged at the rate of 300 rubles per hour, with the exchange being 1 ruble $=\$ 1.10$. Fifty rubles per hour is charged to those using their own M-220.

\section{EDP Merger}

General Electric Co. and Honeywell, Inc., announced that an agreement in principle had been reached in which GE would sell its unprofitable computer operations to Honeywell, Inc. Under terms of the proposed agreement, a new Honeywell subsidiary would be set up to acquire the GE computer operations. If the merger is approved, Honeywell will jump ahead of Univac to hold the No. 2 spot in the computer market, following IBM.

Tapes for Psychophysical Demonstrations

A tape recording entitled "Stimuli for frequency discrimination by the method of constant stimuli," together with 
companion materials, enables the instructor to conduct a psychophysical experiment that yields instructive and reliable data. Applications range from demonstrations in introductory courses to laboratory units in experimental courses. Data may be gathered from large groups through the tape recorder's speaker or from smaller groups using audio distribution techniques.

The tape can be played on any four-track (stereo) recorder taking a 7 -in. reel at $7 \frac{1}{2} \mathrm{in}$./sec. All the data may be gathered for both standard and comparison in less than $20 \mathrm{~min}$. Two versions are available. One contains comparisons that differ by $2 \mathrm{~Hz}$; the other contains $3-\mathrm{Hz}$ differences.

Contents include (1) tone sequences enabling the instructor to anchor the sensation level of the recording at 20,30 , 40 , or $50 \mathrm{~dB}$ re $.0002 \mathrm{dynes} / \mathrm{cm}^{2}$, (2) verbal instructions for judging tone pairs, (3) 70 tone pairs with the standards presented first, (4) verbal instructions, comparison tone first, and (5) 70 tone pairs with the comparison presented first.

Price of the tape recording is $\$ 15$ for either version. A package of 100 data sheets is $\$ 1.50,20$ scoring sheets are $\$ 1.00$, and a booklet, "Guide," sells for $\$ 7.50$ per 20. The booklet provides instructions for analyzing the data. The student is guided through simple graphic methods, the average $\mathrm{Z}$-score method, and an algebraic method for computing DLs and PSEs. Time-order error is discussed.

The distributor has permission from most textbook publishers to reproduce figures, tables, and other portions of books for educational purposes. If one has graphs or drawings that might be incorporated into lectures, $2 \times 2$ slides of the material will be provided on request at $\$ 0.75$ each (minimum order of eight).

The company is relatively new and appears eager to receive suggestions and to discuss the possibility of participation by experimental psychologists in designing and developing instructional materials.

Life Science Associates

P.O. Box 163

Baldwin, New York 11510

\section{Notes}

\section{A well-regulated constant-current shocker ${ }^{1}$}

JOHN BINTZ, UNIVERSITY OF CALIFORNIA, DAVIS, California 95616

Reliably delivering a specified amount of electrical energy to a rodent on a grid floor has long been a problem of both practical

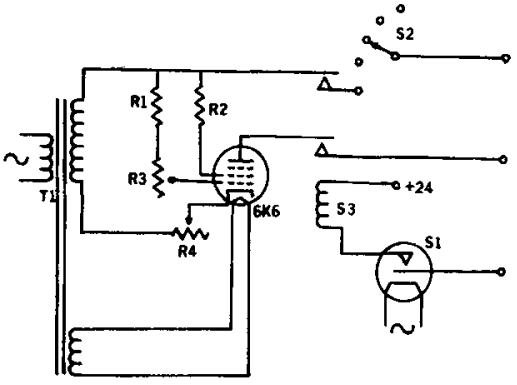

Fig. 1. Shocking circuit that provides well-regulated current to S. Parts list: R 1 10-megohm resistor; R2 - 3.9-megohm resistor; R3, R4 - 1-megohm resistors; T1 - transformer, Stancor P-8179, or equivalent; S1 - Amperite time delay relay, 115N060; S2 - 15-position rotary switch with NE-2s between each position; $S 3-24 V$ relay with high-voltage contacts.

and theoretical importance to psychologists. The simplest and most economical means is to connect even-numbered bars to one side of the voltage source and odd-numbered bars to the other side. When this method is used, two problems are encountered: a fetal boli across any two adjacent bars provides a sufficiently low resistance to short the entire grid so that $S$ receives no shock; in addition, $S$ may learn to stand only on grids of the same polarity and receive no shock.

The most common solution to these problems is the scrambling device that continually changes the polarity of each bar with respect to others. This may be done with a stepping switch, a magnet rotating within a configuration of reed relays, a rotating disk with holes that operate photocell relays, a system of pulsed flip-flops, or by other means. But scrambling circuits have at least two drawbacks, and most have additional disadvantages which apply to some but not all such devices. One lead is required for each and every bar, which generally means a separate cable is required to carry shock. In some situations, e.g., in the two-compartment shuttlebox in which the E wishes to isolate shock to each compartment, a large number of switching contacts are required to control the stimulus. Also, the output of any scrambler

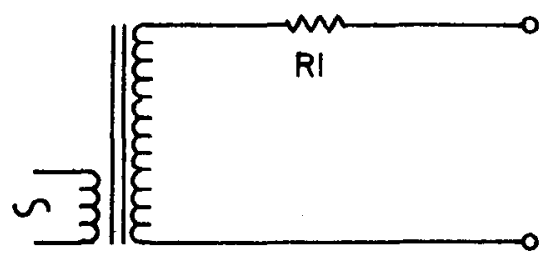

Fig. 2. Simple shocking circuit that requires no scrambler and provides fair regulation. is a series of spikes, which may in itself have undesirable effects. The rate of these spikes, and consequently the amount of shock received, depends upon the number of grids $\mathbf{S}$ is in contact with. There are, probably. systematic differences in posturing by $S s$, and therefore increased inter-S variability. Some shocking devices offer additional disadvantages, such as high cost, excessive noise, low switching rate, inadequate or differing shock "on" time between different grid bars, and wide fluctuation in the current actually delivered to S.

The shocking device described herc has been used to eliminate most of these problems. This device is based on the operating characteristics of the NE-2 neon bulb, i.e., the bulb acts as a conductor whenever the voltage is above a breakover voltage and as an infinite resistance when the voltage is below that level. One of the bulbs is connected between each of the grid bars, and a voltage is impressed to the entire series. This enables current to flow through the bulbs when no $S$ is in the system. However, when $S$ is between any two of the bars, the parallel resistance across the neon bulb drops the voltage below the breakover point, the bulb stops conducting, and the current is diverted through S. Only two leads are required, one to each end of the string of neom bulbs. This principle has been presented before by Brown, Reus, and Webb (1961), whose circuit provides the basis for the method presented here. Unfortunately, in two attempts we were unable to operationalize the Brown et al circuit with the specified components. Also, adjustment for differing numbers of grid bars is made by switching transformers in series, a bulky and expensive method of control, and one lacking in precision.

The circuit diagram for the shocking device used in our laboratory is presented in Fig. 1. The major active element in the circuit is the pentode (6K6), which provides half-wave rectification in addition to constant current. It does so despite large load changes or changes in the number of grid bars contacted or spanned by $\mathrm{S}$. The pentode requires about $1 \mathrm{~min}$ of warm-up time and is guaranteed by the thermal relay (S1). Current level control is provided by R3 and R4, where R3 is the course and R4 the fine adjustments. $S 2$ is a 15-position rotary switch with NE-2s wired across each position. The switch enables $E$ to dial the number of grid bars in the experimental environment and maintain exactly the same electrical parameters in two environments differing in the number of bars. $\mathrm{S} 3$ is a control relay operated at times when shock is to be delivered.

It is possible to use the neon bulb principle in a far simpler circuit such as 